difference in demographics and complication (distal emboli, mortality rate, symptomatic intracranial hemorrhage). The mean time from onset to groin puncture, successful reperfusion rate (defined thrombolysis in cerebral infarction $2 b / 3$ ) and favorable functional outcome (determined by modified Rankin Scale score 0-2 at 90 days) also showed no significant statistically difference. But MT group had a significantly shorter mean procedure time (45.84 vs. $76.06 \mathrm{~min} ; \mathrm{p}=$ $0.001)$ and hospital stay (11.53 vs. 15.0 day; $\mathrm{p}=0.042)$, and a lower 1-day NIHSS score (6.35 vs $10.88 ; \mathrm{p}=0.012)$.

Conclusion In ineligible patient for IVT, MT alone isn't inferior to MT combined with IVT.

Disclosures J. Sung: None. M. Lee: None. D. Lee: None. H. Lee: None. S. Yang: None.

\section{E-107 SHORT-TERM IN-HOSPITAL OUTCOMES OF THROMBOLYSIS FOR ACUTE ISCHEMIC STROKE PATIENTS WITH NON-PRIMARY BRAIN TUMORS AND HYPERTENSION}

K Tong*, A Wang-Selfridge, P Brauer. Anatomy, Kansas City University of Medicine and Biosciences, Kansas City, MO

\subsection{6/neurintsurg-2019-SNIS.182}

Background Intravenous thrombolysis remains an underutilized treatment for acute ischemic stroke (AIS) due to several relative and absolute contraindications. Previous studies have found similar outcomes after thrombolysis between AIS patients with benign brain tumors and AIS patients without. This study aims to investigate short-term outcomes of thrombolytic treatment for the greater majority of AIS patients who have no history of primary brain tumors, particularly those with hypertension (HTN).

Methods This retrospective cohort study utilized data from the 2012-2015Q3 Nationwide Inpatient Sample (NIS). ICD-9 codes identified adult patients (ages 18+) who suffered acute ischemic stroke and received intravenous thrombolysis, and then further isolated patients diagnosed with HTN. Data for patients who were missing important clinical identifiers (age, gender, race, mortality), did not receive IV thrombolysis, and had primary brain tumors (benign or malignant) were excluded. Data analyses assessed hospital mortality rate, length of stay (LOS), inpatient charges, and average age of admission.
Results Of the 24,692 encounters with AIS patients treated with thrombolysis and had no history of primary brain tumors, 15,219 were diagnosed with HTN.

- Mean mortality rate was significantly decreased (6.3\% HTN vs. $8.1 \%$ no HTN, $\mathrm{p}<0.0001)$.

- Mean LOS was significantly shorter (5.90 days HTN vs. 7.05 days no HTN, p <0.0001).

- Average total charges were significantly decreased (\$81,467.75 HTN vs. \$93,538.04 no HTN, p<0.0001).

- Average age at admission was significantly older (67.73 years HTN vs. 65.95 years no HTN, p<0.0001).

Conclusion This study aims to inform physicians to better manage acute ischemic stroke patients receiving IV thrombolysis with hypertension and no history of primary brain tumors. These patients experience lower mortality rate, shorter LOS, decreased total hospital charges, and older age at admission than those without HTN. The mortality rate and LOS could be explained by a wider range of care provided from an interdisciplinary healthcare team, as opposed to simple post-op observational care. Future research should aim to delineate the differences in approach to determine which aspects improve outcomes for AIS patients with hypertension who receive intravenous thrombolysis.

Disclosures K. Tong: None. A. Wang-Selfridge: None. P. Brauer: None.

\section{E-108 INCIDENCE OF IN-SITU THROMBOSIS IN ACUTE LARGE VESSEL OCCLUSION: REVIEW OF 168 THROMBECTOMY PATIENTS}

M Alexander* , Z Barnard. Department of Neurosurgery, Cedars-Sinai Neurovascular Center, Los Angeles, CA

\subsection{6/neurintsurg-2019-SNIS. 183}

Introduction Acute large vessel occlusion (LVO) presenting with stroke symptoms may occur as a result of embolic occlusion, in-situ thrombosis of intracranial atherosclerotic stenosis or intracranial arterial dissection, vasculopathy, or other etiologies. The literature reflects variation in the incidence of these pathologies depending on geography, heredity, smoking history, as well as others factors.

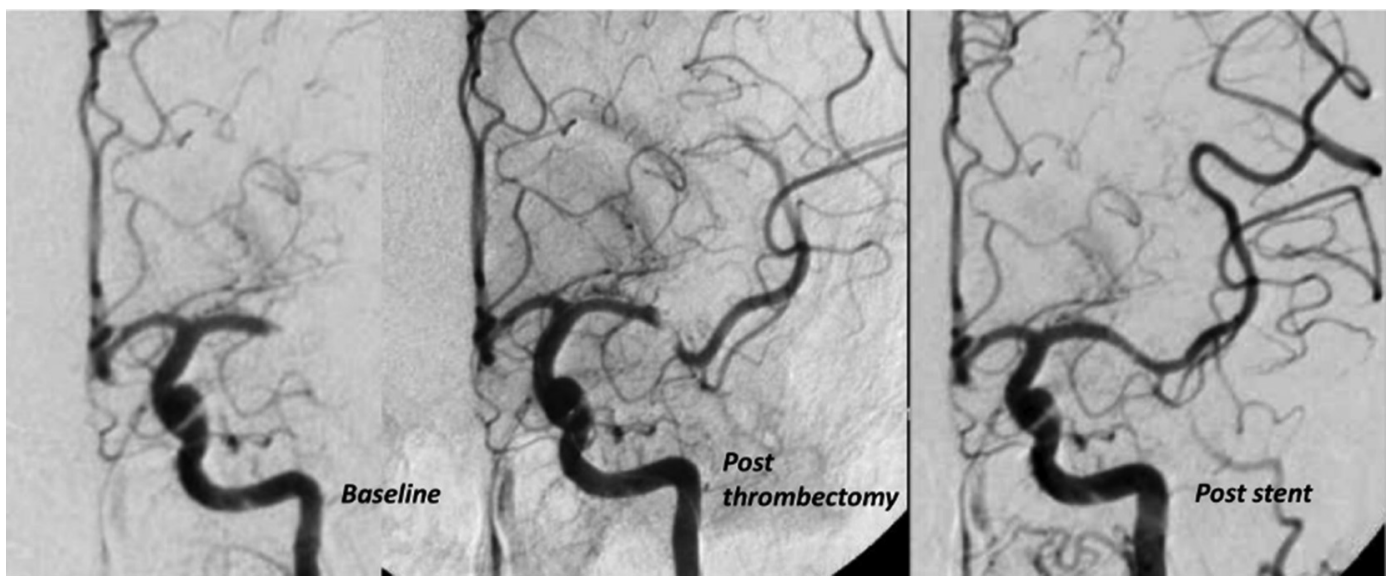


Methods We retrospectively analyzed a consecutive series of 168 patients who presented with acute stroke symptoms and imaging revealing intracranial large vessel occlusion, who underwent cerebral angiography and attempted endovascular thrombectomy. Patients who underwent successful thrombectomy with no evidence of post-thrombectomy underlying ICAD lesion, dissection, or other vascular abnormality were presumed to have an embolic cause of their LVO. Patients who had an underlying vessel abnormality post-thrombectomy (exclusive of mechanical vasospasm) and no history of atrial fibrillation or other embolic risk factor were categorized as insituthrombosis.

Results Of the 168 patients, 151 arteries were revascularized with no post-thrombectomy vessel abnormality, indicating potential embolic cause. The remaining 17 patients had an underlying abnormality of which 15 appeared to be atherosclerotic in nature and 2 with underlying arterial dissection. These patients were initially indentified by the inability to pass the occlusive lesion with a J shape microwire. All 17 patients underwent angioplasty of their stenotic lesion at the time of thrombectomy. In addition, 13 patients underwent intracranial stenting. This included 8 patients stented acutely and 5 patients stented in a delayed fashion. Stenting was typically performed acutely if there was significant arterial recoil or lack of sufficient flow following angioplasty alone. One patient appeared to have an acute stent thrombosis within 12 hours of stenting.

Conclusions Although the majority of acute LVO stroke was embolic in this series, $10.1 \%$ of patients appeared to have a clinical picture more consistent with in-situthrombosis and $8.9 \%$ of the total patient cohort appeared to have an underlying ICAD lesion. Within this regional series, in-situ occlusion is a less common, but significant pathology, that may require different treatment strategies that conventional thrombectomy for LVO.

Disclosures M. Alexander: 2; C; Stryker Neurovascular, Medtronic, Penumbra, Inc. Z. Barnard: None.

\section{E-109 SHORT-TERM IN-HOSPITAL OUTCOMES OF THROMBOLYSIS FOR ACUTE ISCHEMIC STROKE PATIENTS WITH NON-PRIMARY BRAIN TUMORS AND ELECTROLYTE IMBALANCES}

K Tong*, A Wang-Selfridge, P Brauer. Anatomy, Kansas City University of Medicine and Biosciences, Kansas City, MO

\subsection{6/neurintsurg-2019-SNIS. 184}

Background Intravenous thrombolysis remains an underutilized treatment for acute ischemic stroke (AIS) due to several relative and absolute contraindications. Previous studies have found similar outcomes after thrombolysis between AIS patients with benign brain tumors and AIS patients without. This study aims to investigate short-term outcomes of thrombolytic treatment for the greater majority of AIS patients who have no history of primary brain tumors, particularly those with electrolyte imbalances.

Methods This retrospective cohort study utilized data from the 2012-2015Q3 Nationwide Inpatient Sample (NIS). ICD-9 codes identified adult patients (ages $18+$ ) who suffered acute ischemic stroke and received intravenous thrombolysis, and then further isolated patients diagnosed with electrolyte imbalances. Data for patients who were missing important clinical identifiers (age, gender, race, mortality), did not receive IV thrombolysis, and had primary brain tumors (benign or malignant) were excluded. Data analyses assessed hospital mortality rate, length of stay (LOS), inpatient charges, and average age of admission.

Results Of the 24692 encounters with AIS patients treated with thrombolysis and had no history of primary brain tumors, 5450were diagnosed with electrolyte imbalances (EI).

- Mean mortality rate of patients was significantly increased (10.4\% with EI vs. $6.0 \%$ with no EI, $\mathrm{p}<0.0001)$.

- Mean LOS was significantly longer (9.21 days with EI vs. 5.53 days with no EI, $\mathrm{p}<0.0001)$.

- Average total charges were significantly increased $(\$ 117,906.49$ with EI vs. $\$ 77,032.77$ with no EI, $\mathrm{p}<0.0001)$.

- Average age at admission was significantly older (67.88 years with EI vs. 66.81 years with no EI, p<0.0001)

Conclusion This study aims to inform physicians to better manage AIS patients receiving IV thrombolysis with electrolyte imbalances and no history of primary brain tumors. These patients experience higher mortality rate, longer LOS, increased total hospital charges, and older age at admission than those without EI. These findings suggest that placing clinical focus on managing electrolyte imbalances before administering IV thrombolysis for AIS may be critical for improving short-term in-hospital outcomes. Future research should aim to investigate different thrombolytic agents to determine the most optimal choice for patients with electrolyte imbalances.

Disclosures K. Tong: None. A. Wang-Selfridge: None. P. Brauer: None.

\section{E-110 ENDOVASCULAR TREATMENT DECISION IN ACUTE STROKE: DOES PHYSICIAN SEX MATTER? INSIGHTS FROM AN INTERNATIONAL MULTIDISCIPLINARY SURVEY}

${ }^{1}$ J Ospel, ${ }^{2} \mathrm{~N}$ Kashani, ${ }^{3} \mathrm{~B}$ Campbell, ${ }^{4} \mathrm{M}$ Foss, ${ }^{5} \mathrm{~F}$ Turjman, ${ }^{6} \mathrm{~S}$ Yoshimura, ${ }^{7} \mathrm{~A}$ Wilson, ${ }^{8} \mathrm{~W}$ Kunz, ${ }^{9} \mathrm{M}$ Cherian, ${ }^{10} \mathrm{~B}$ Kim, ${ }^{11} \mathrm{~A}$ Rabinstein, ${ }^{12} \mathrm{U}$ Fischer, ${ }^{13} \mathrm{P}$ Sylaja, ${ }^{14} \mathrm{~B}$ Baxter, ${ }^{15} \mathrm{~J} \mathrm{Heo}$, ${ }^{4} \mathrm{~B}$ Menon, ${ }^{16} \mathrm{G}$ Saposnik, ${ }^{4} \mathrm{M}$ Hill, ${ }^{17} \mathrm{M}$ Goyal ${ }^{*},{ }^{7} \mathrm{M}$ Almekhlafi. ${ }^{1}$ University Hospital Basel, Basel, Switzerland; ${ }^{2}$ Radiology, University of Calgary, Calgary, $A B$, Canada; ${ }^{3}$ Royal Melbourne Hospital, Melbourne, Australia; ${ }^{4}$ University of Calgary, Calgary, AB, Canada; ${ }^{5}$ Centre Hospitalier Universitaire de Lyon, Lyon, France; ${ }^{6}$ Neurosurgery, Hyogo College of Medicine, Hyogo, Japan; ${ }^{7}$ Radiology, Cumming School of Medicine, University of Calgary, Calgary, AB, Canada; ${ }^{8}$ Radiology, University Hospital, LMU Munich, Germany; ${ }^{9}$ Koval Medical center, Tamil Nadu, India; ${ }^{10}$ St. Mary's Hospital Seoul, Seoul, Korea, republic of ${ }^{11}$ Radiology, Mayo Clinic Rochester, Rochester, MN; ${ }^{12}$ University Hospital Bern, Bern, Switzerland; ${ }^{13}$ Sree Chitra Tirunal Institute for Medical Sciences and Technology, Thiruvanthapuram, India; ${ }^{14}$ Erlanger Medical Center, Chattanooga, $T N_{i}{ }^{15}$ Yonsei University, Seoul, Seoul, Korea, republic of; ${ }^{16}$ University of Toronto, Toronto, ON, Canada; ${ }^{17}$ Cumming School of Medicine, University of Calgary, Calgary, AB, Canada

\subsection{6/neurintsurg-2019-SNIS.185}

Introduction Numerous studies have compared treatment approaches of female and male physicians in different medical subspecialties, some of them revealed significant differences. To date, only few women are engaged in the neurointerventional field. Hence, it is unclear whether the treatment practice is influenced by physicians' sex. We explored whether there are differences in treatment decisions made by female and male physicians.

Materials and methods An international cross-sectional survey of both female and male stroke physicians and neurointerventionalists was conducted. Participants were randomly assigned 